\title{
Prognostic importance of the nature of the course of pregnancy and the dental status of their children
}

Keywords: quality, complications, gestation, pregnancy, toxicosis

\section{Opinion}

Tooth decay reduces the quality of life, which occurs for a number of reasons. Teeth are an important component of full speech, active verbal communication, and communicative skills at any age, and, consequently, the fullness of mental and behavioral reactions. Quality of life is especially impaired when a child has large numbers of teeth (active dental caries), and often these are complicated forms of caries. In this case, the child is often and for a long time sick - he or she suffers from physical suffering and the parent takes a sick leave to care for the child. Also in the case of multiple tooth decay, replacement of existing fillings and repeated treatment of affected teeth are much more frequently required. Predicting and timely correction of such conditions will help avoid complications and improve the oral health of future generations.

In order to be able to predict the health status of children, depending on the nature of the course of pregnancy, 169 couples were examined and observed during the whole period of gestation. The dental health of 172 children born to them was monitored from birth to 3 years. When conducting a correlated analysis of the relationship between the nature of the course of pregnancy and the dental health of the child, statistically significant interrelationships of these indicators have been revealed. Moreover, when calculating the gamma coefficient, the data on the most significant results in correlation of the physiological course of toxicosis and absence of dental pathology were obtained $(\mathrm{Gamma}=0.90)$. From this we can conclude that there is a high probability of a physiological course of pregnancy in a woman with no history of somatic pathology.

Gamma factor is the most significant factor in correlation of physiological course of toxicosis and caries resistance of child's hard tissues (Gamma=0.96). The likelihood that the mother's oral hard tissue will be caries resistant in a combined pregnancy is significantly low (Gamma=-0.72 - inverse strong correlation). The most significant effect on the presence of decompensated flow of caries in the child is late toxicity of pregnancy $(\mathrm{Gamma}=0.69)$. When used for statistical processing of these coefficients Kandel-Tau and Spearman revealed statistically significant differences in the effect of the nature of the course of pregnancy of the mother on the condition of dental hard tissues in the physiological course $(r=0.59)$ and in the combined toxicosis $(\mathrm{r}=0.38)$. Thus, in the physiological course of pregnancy with a high probability $(96 \%)$ it is possible to predict caries resistance of the hard tissues of the oral cavity of the child, while this possibility in any form of toxicosis is virtually ruled out.

In studying the relationship between the degree of caries activity in caries-exposed children from the nature of the course of pregnancy, the results obtained: in the physiological course of pregnancy, the birth of children with caries resistant hard tissues of the oral cavity is much higher than in any type of toxicosis. Of the children surveyed, only 6 (3.5\%, MDIs: $1.3-7.5 \%)$ were caries resistant, three of them were born to mothers with physiological course of pregnancy, while others were born to mothers with late toxemia (so out of 158 children born
Volume 9 Issue I - 2020

\author{
IMVoloshina \\ Department of Therapeutic Dentistry, Russian Ministry of \\ Health, Russia
}

Correspondence: IMVoloshina, Candidate of Medical Sciences, Associate Professor of the Department of Therapeutic Dentistry, FSBOUVOO OmGMU Russian Ministry of Health, Russia, Email 25082000.80@mail.ru

Received: January 18, 2020 | Published: January 27, 2020

to mothers suffering from toxemia, only $1.9 \%$ were caries resistant). The condition of hard tissues of the mouth cavity of children born from physiologically proceeding pregnancy is authentically better: $27,3 \%$ of persons are caries resistant and $15,2 \%$ have the compensated current of caries - in case of toxicosis of pregnancy only $3.2 \%$ are caries resistant and $16,8 \%$ have the compensated current. In the physiological course of pregnancy, the mother is significantly more likely to give birth to caries-resistant children (Gamma coefficient 0.96-strong correlation). Not a single carious-resistant child from mothers with early gestational toxicosis has been identified. 96.5 per cent (MDIs: 92.5-98.7 per cent) of children are affected by caries, most of them from mothers with toxicosis of pregnancy $(\mathrm{p}<0.05)$. For all types of toxicosis, the decompensated flow of caries is most frequently detected $(63.9 \%, 52.4 \%$ and $71.4 \%$, respectively). At early toxicity of pregnancy and at non-physiological course of the whole period of gestation, the mother is significantly more likely to give birth to children who will have decompensated course of caries (Gamma coefficient 0.5 -average correlation relation and 0.7 -average correlation relation, respectively). In early toxicosis, a compensated flow is least likely to be detected $(14.7 \%)$. In late toxemia of pregnancy in the mother, the highest correlation relation was found with a sub compensated course of caries (Gamma coefficient 0.7 average correlation relation).

Statistical analysis of the results obtained (general discriminant analysis was performed) showed that the presence of pregnancy toxicity in the mother is a predictor of caries susceptibility in children $(\mathrm{x} 2=39.5, \mathrm{p}=0.0000)$. At the same time, $91.9 \%$ of children have a correct prognosis of caries susceptibility in the presence of toxicosis in the mother: in the absence of toxicosis, intact oral cavity will be detected with $95.6 \%$ reliability.

Thus, the physiological course of pregnancy can serve as a prognostic sign of caries resistance of the child's hard tissue with a high probability. The predictor of the condition of children's oral hard tissues within the framework of the material under study is precisely the combined toxicosis in mothers-the correctness of the forecast in $69.2 \%$ of cases. This indicator can be used as a prognostic factor.

\section{Acknowledgments}

None. 


\section{Conflicts of interest}

The author declares that there are no conflicts of interest.

\section{Funding}

None.

\section{References}

1. Abrosimova ON. Ballistic estimation of treatment quality of the complicated forms of temporary teeth caries in children in the nearest and distant future (in Russian). System analysis and control in biomedical systems. 2013;12(4):1141-1144.

2. Budaichiev MG. Medico-social aspects of dental morbidity among children: (based on materials of the Republic of Dagestan). Cand of Medical Sciences. Moscow:2012:23.

3. LP Kiselnikova. Influence of tooth decay intensity and hygienic state of the mouth cavity on life quality of children of 3-6 years old. Institute of Dentistry. 2012;1(54):28-30.

4. EA. Voevoda, IN Golubeva, EI Ostapko. Features of saliva mineralizing function in children with different degree of dental caries activity. Modern dentistry. 2014;1(58):79-80.

5. Danilova MA, Yu Shevtsova. Risk factors of early childhood caries development (in Russian). Modern problems of science and education. 2014;(4):300.
6. Zykin AG. Optimization of the prophylactic methods of the basic dental diseases at the primary school age children and teenagers (in Russian). Problems of dentistry. 2014;(3):54-56.

7. Ermakova LA, Nagorny SA, Krivorotova EY, et al. Dirofilaria repens in the Russian Federation: current epidemiology, diagnosis, and treatment from a federal reference center perspective. Int J Infect Dis. 2014;23:47-52.

8. Kirillova EB. Improvement of methods of treatment and prevention of tooth decay in children of early age: authored. Candidate of Medical Sciences. Moscow: 2013:24.

9. Leontief VK, VV Kolpakov, AV Bragin. Concept of Typical Variability of a Physiological Individuality--A Fundamental Basis of Systemic Prevention and Combined Treatment in Stomatology. Stomatologiia. 2005;84(5):4-9.

10. Leontief VK, GN Pakhomov. Dental diseases prevention (in Russian). Moscow: KMK-Invest. 2007:700.

11. Namrata Patil, Shantanu Choudhari, Sadanand Kulkarni. Comparative evaluation of remineralizing potential of three agents on artificially demineralized human enamel: an in vitro study. J Conserv Dent. 2013; 16(2):116-120.

12. A Kensche, M Reich, K Kümmerer, et al. Lipids in preventive dentistry. Clin Oral Investig. 2013;17(3):669-685. 